Elsevier Editorial System(tm) for Journal of

Electron Spectroscopy and Related Phenomena

Manuscript Draft

Manuscript Number: ELSPEC-D-07-00063R1

Title: High-resolution KLM Auger spectra of Ni metal excited by X-rays

Article Type: Regular Article

Keywords: Auger spectra; Ni KLM; synchrotron radiation; intrinsic excitations; transition energy; relative intensity; 3d transition metals

Corresponding Author: Mr Sandor Egri,

Corresponding Author's Institution: University of Debrecen

First Author: Sandor Egri

Order of Authors: Sandor Egri; Laszlo Kover; Wolfgang Drube; Istvan Cserny; Mihaly Novak

Abstract: KLM Auger spectra of $\mathrm{Ni}$ metal were measured with high energy resolution and high statistical accuracy using monochromatic synchrotron radiation. The spectra were corrected for the background from inelastically scattered electrons using Partial Intensity Analysis where the electron trajectories were calculated by Monte Carlo simulation. Following background correction, the corrected spectra were fitted to model peaks taking into account intrinsic excitations. The measured transition energy of the most intense KL2M3 (1D2) Auger line is 7388.1 (+/- 0.4) eV. The obtained relative intensities and energies of the Auger diagram lines are compared to published calculations as well as to experimental data for other $3 d$ transition metals. In addition, the presence of $\mathrm{Ni} 3 \mathrm{~s}, 3 \mathrm{p}$ photoelectron peaks in the spectra excited internally by $\mathrm{Ni}$ Ka X-rays is shown. 


\section{Dear Professor Pireaux!}

Here attached I send you the revised version of the manuscript. (MS. No.:ELSPEC-D-0700063)

In the cover letter changes are summerized, I also included detailed form of them in two additional files as „Detailed response to Reviewers”.

The way of the presentation of the data has been changed in all of the Tables according to the comments of both Reviewers.

Decimal points have been used to improve the clarity.

- For the clarity the decimal point is used in all of the error data of the tables. Instead of 184.4(1), 137.9(1) etc. 184.4(1.0), 137.9(1.0) etc. and the footnote about the notation of the errors has been changed accordingly. - Each data with hundredths of eV were presented have made rounded to tenths of eV.

- The notation for Table 2 has been changed "in eV" included. (comment 2a)

-In the case of $\mathrm{Fe}$ and $\mathrm{Ni}$ the unambigous notation has been changed in Table 2. In Fe from 165.0 (9) to 165.0 (0.9), from 123.5 (8) to 123.5 (0.8) etc, and in $\mathrm{Ni}$ from $184.4(1)$ to $184.4(1.0)$ etc.

-In the case of Cr in relative energy data deviations from ref [5] has been found and corrected.

-In Table $326(4)$ has been changed into 26(4.0), from 52(8) to 52(8.0) etc. -In the Table 4 in the coloumn of $\mathrm{Fe}$ and $\mathrm{Ni}$ also the decimal point has been used.

-In table 5 in row for KL3M23 from 4 (0.2) to 4.0 (0.2) and in row for

KL3M45 from $6(0.4)$ to $6.0(0.4)$ (comment 1,2 b)

According to the suggestions and comments of Reviewer \#1 chapter „Results and discussion” has been changed slightly (some words deleted, some are added).

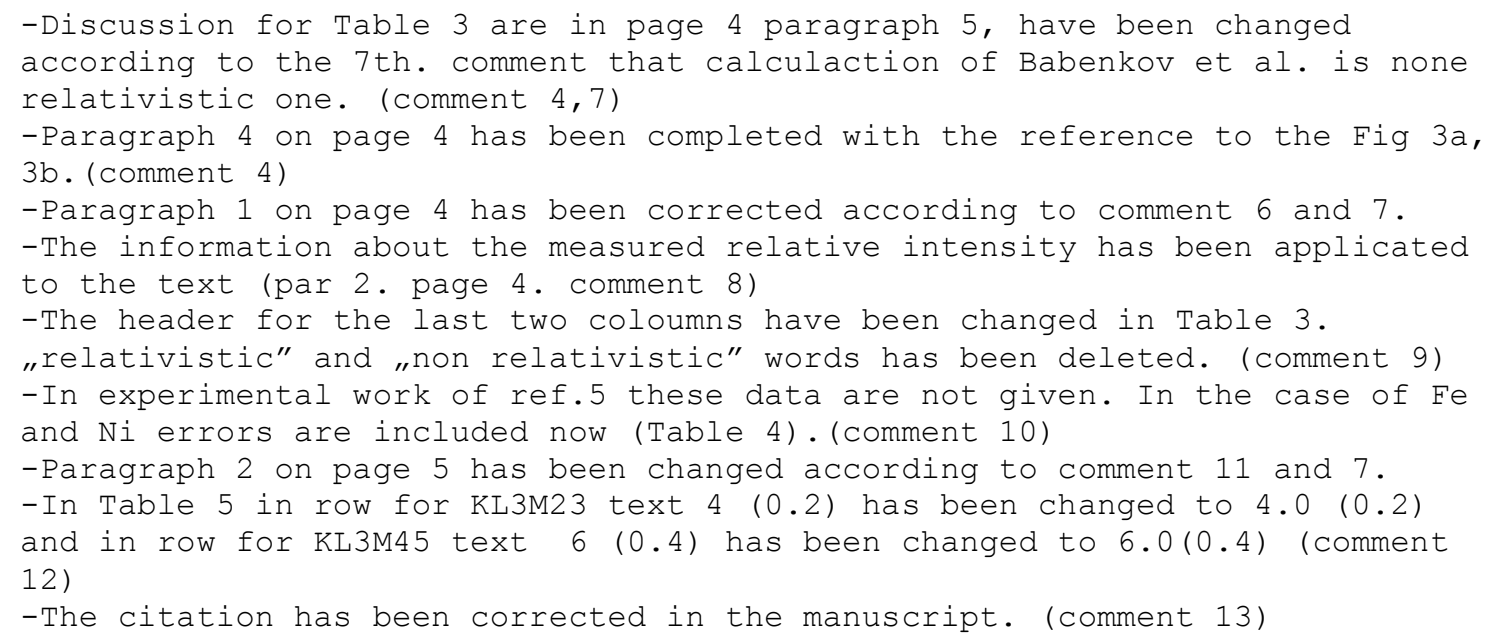

I hope that revised version of the manuscript will now be suitable for publication. Looking forward to hearing from you, with my best regards

Sandor Egri 
Response to the comments of Rewiever \#1

Thank you for your valuable comments and recommendations.

Detailed replay to the comments:

1) Table $1 \mathrm{a}$

A notation 184.4(1) (footnote c)) usually means 184.40 .1$.

According to the footnote $c$ ), the measured relative energy of 69.77(2) for the KLIM4,5 transition should be read as 69.77 2.00. What is then the sense to determine the energy with such a high precision (hundredths of eV) when the standard deviation quoted is by 3 orders of magnitude higher? This remark also concerns about the transitions KL2M1(42.37(1.5)), KL2M2(5.77(2)), and KL2M4, $5(68.83(1))$.

- For the clarity the decimal point is used in all of the error data of the tables. Instead of 184.4(1), 137.9(1) etc. 184.4(1.0), 137.9(1.0) etc. and the footnote about the notation of the errors has been changed accordingly. From $^{\mathrm{c}}-184.4(1)$ is a short notation for $-184.4 \pm 1.0$ to ${ }^{\mathrm{c}}-184.4(1.0)$ is a short notation for $-184.4 \pm 1.0$

- Each data with hundredths of eV have made rounded to tenths of eV.

\section{2) Table 2}

a) There is no information on energy units in which transition energies are quoted.

- The notation for Table 2 has been changed "(in eV)" has been included.

b) Relative energies for $V, C r$, and Mn are presented in an understandable way. However, this is not the case of Fe and Ni. Has one to read data for Fe $165.0(9), 123.5(8)$, and $63.5(12)$ as $(165.0$ 9.0), (123.5 8.0), and (63.5 12.0), respectively? Is accuracy of these data (Ref. 4) so poor? Similar doubts arise in reading of the experimental $N i$ KL3MI transition intensity in Table 3 and Table 4. Presentation of experimental energy widths for the KL3M2,3 and KL3M4,5 transitions is also unusual.

Summary: I highly recommend using the same and understandable notation of experimental data for all tables in the paper.

- In the case of $\mathrm{Fe}$ and $\mathrm{Ni}$ the unambigous notation has been changed in Table 2 . In $\mathrm{Fe}$ from 165.0 (9) to 165.0 (0.9), from 123.5 (8) to 123.5 (0.8) etc, and in $\mathrm{Ni}$ from $184.4(1)$ to $184.4(1.0)$ etc.

-In the case of Cr in relative energy data deviations from ref [5] has been found and corrected.

-In Table 3 similarly $26(4)$ has been changed into 26(4.0), from 52(8) to 52(8.0) etc.

-In the Table 4 in the coloumn of $\mathrm{Fe}$ and $\mathrm{Ni}$ also the decimal point has been used.

-In table 5 in row for KL3M23 from 4 (0.2) to 4.0 (0.2) and in row for KL3M45

from $6(0.4)$ to $6.0(0.4)$ 


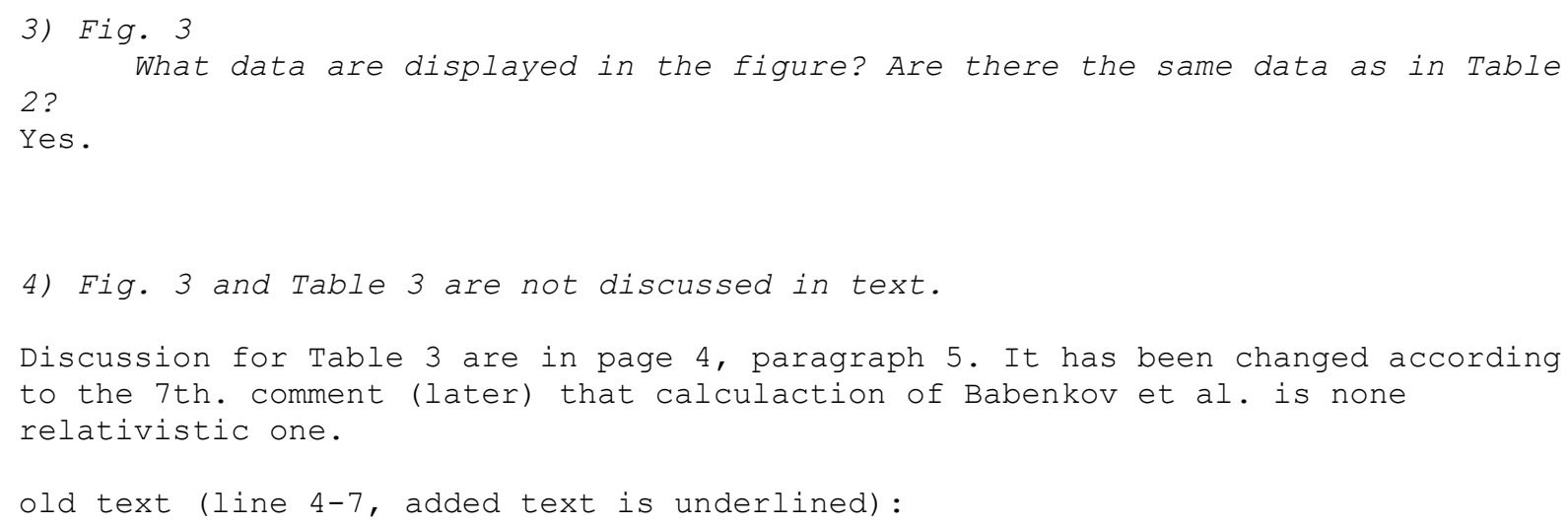

...the relativistic intermediate coupling model. In addition, the former model predicts that this transition is two times more intense than the reference $\mathrm{KL}_{2} \mathrm{M}_{23}$ transition (Table 3). The present experimental data (Table 3 ) are in a good agreement with the relativistic intermediate coupling model ${ }^{20}$, confirming its validity for the Ni KLM Auger transitions.

New version:

..the intermediate coupling model presented in Ref. 20. In addition, the former model predicts that this transition is two times more intense than the reference $\mathrm{KL}_{2} \mathrm{M}_{23}$ transition (Table 3). The present experimental data (Table 3) are in a good agreement with the intermediate coupling model calculations of Babenkov et al. ${ }^{20}$, confirming its validity for the Ni KLM Auger transitions.

Paragraph 4 on page 4 has been completed with the reference to the Fig 3a, 3b.

6) $p$ (page) 4, I (Iine) - 1

Only 11 spectrum components were resolved in the KLM Auger spectrum of Ni

- not 13 as stated in the paper. Additional 2 lines are connected with spectrum excitation.

It has been corrected in the text.

Old:

For the assignment of these components we used published theoretical calculations of the energy positions and the relative intensities of the Ni KLM diagram lines.

New:

11 of them belong to the KLM Auger spectrum and 2 additional lines are connected with the spectrum excitation. For the assignment of components of KLM Auger spectrum we used published theoretical calculations of the energy positions and the relative intensities of the Ni KLM diagram lines.

7) $p 4,1-7$

The calculations of the KLM transition rates by Babenkov et al. [20] are

non-relativistic ones.

Old:

Relativistic calculations for intensities in intermediate coupling scheme were published by Babenkov et $\mathrm{al}^{20}$. Similar calculations were made by Chen at al. but in the $\mathrm{jj}$ coupling scheme $\mathrm{e}^{21}$.

New:

Non-relativistic calculations for intensities in intermediate coupling scheme were published also by

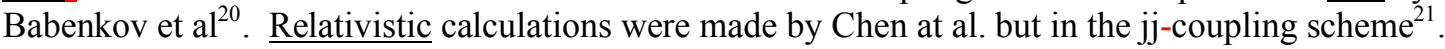

It has been corrected in Table 3 and in the notation for Table 4 also.

8) $p 4,1-13$ 


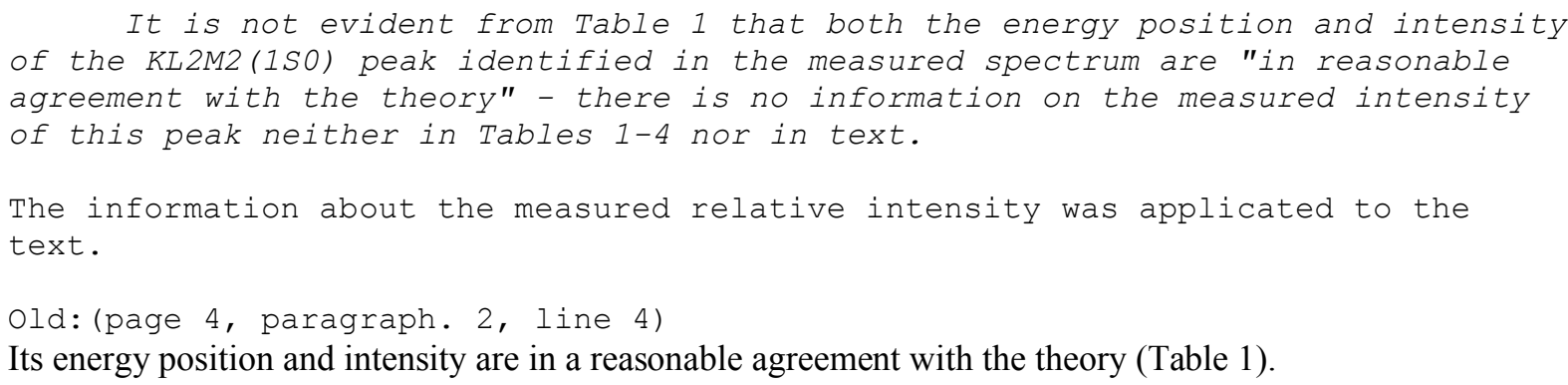

Its energy position and measured relative intensity of 9.2 to the $1 \mathrm{D} 2$ peak are in a reasonable agreement with the theory (Table 1a)

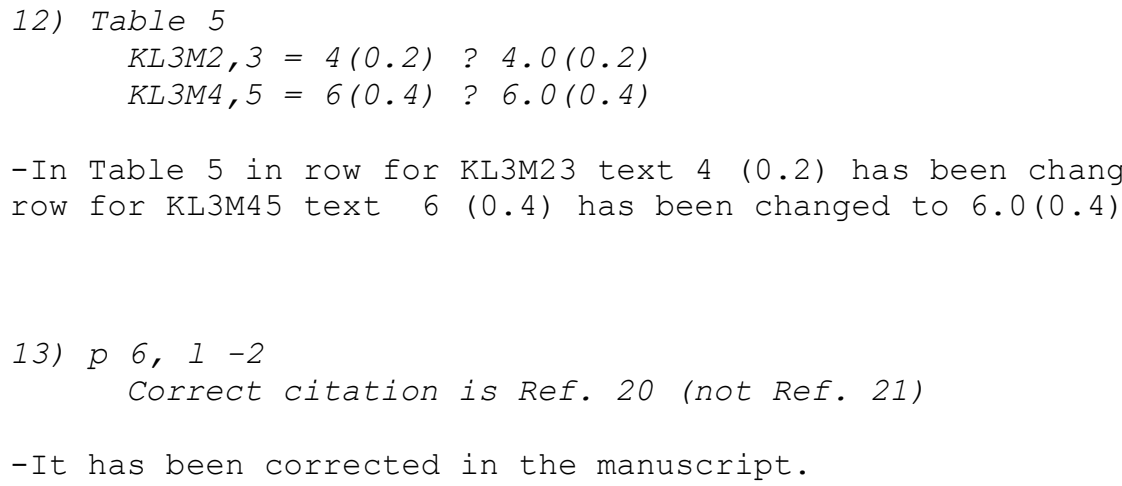




\author{
Response to the comments of Rewiever \#2 \\ Thank you for your valuable comments and recommendations. \\ Detailed replay to the comments:
}

Reviewer \#2: This is a clearly written paper about high resolution measurements of the Ni KLM Auger transitions: at least, it involves a much higher resolution than in previous work on high energy transitions of the $3 \mathrm{~d}$ metals. When combined with the sophisticated background treatment described, we can consider that the paper represents an important advance in this area. It occurs to me that a study comparing the results of various background treatment schemes would be appropriate. What happens when the authors use the same treatment as previous authors? By how much do the Ni KLM results change?

Inelastic background correction is not a critical point in this analysis, because the inelastic background of $\mathrm{Ni}$ are smooth, without sharp plasmon peaks. The sample can regarded as half-infinite, so because of the isotropic emission of Auger-electrons multiplte inelastic scatterings follow simple exponencial decay. In this case Tougaard-type background correction with universal cross section leeds similar result than background correction formula used in this work according to Partial Intensity Analysis.

However the asymmetric intrinsic line shape of the Auger peaks was treated in previous evaluation (Ref. 5) as a part of the Tougaard type inelastic background and this can cause remarkable changes in relative intensities.

The English is acceptable, with few mistakes (e.g., "studies on" instead of "studies of" on p.2).

It has been corrected in the manuscript.

I think that it is suitable for publication, with a few, minor, modifications: the figures should be clearly numbered and the Tables should be checked for consistency (e.g., in Table la, the signs of theoretical and experimental energies are not always consistent).

The signs of several first theoretical data in the Table 1 have been corrected, and many other changes have been made according to the recommendation of Reviewer \#1 in order to improve the clarity and consistency of the presentation of results.

Numbers of the Figures are not appeared in postscript, but they are 1, 2, 3a and $3 \mathrm{~b}$ in the order of the position in the postscript manuscript as in "Figure captions" on page 8 . 


\title{
High-resolution KLM Auger spectra of Ni metal excited by X-rays
}

\author{
S. Egri ${ }^{1,2}$, L. Kövér ${ }^{2}$, W. Drube ${ }^{3}$, I. Cserny ${ }^{2}$ and M. Novák ${ }^{2}$ \\ ${ }^{I}$ Department of Experimental Physics, University of Debrecen, 18/a Bem tér, H-4026 Debrecen, \\ Hungary \\ ${ }^{2}$ Institute of Nuclear Research of the Hungarian Academy of Sciences, 18/c Bem tér, H-4026 \\ Debrecen, Hungary \\ ${ }^{3}$ Hamburger Synchrotronstrahlungslabor am Deutschen Elektronen-Synchrotron DESY, Notkestr. 85, \\ D-22603 Hamburg, Germany
}

\begin{abstract}
KLM Auger spectra of Ni metal were measured with high energy resolution and high statistical accuracy using monochromatic synchrotron radiation. The spectra were corrected for the background from inelastically scattered electrons using Partial Intensity Analysis where the electron trajectories were calculated by Monte Carlo simulation. Following background correction, the corrected spectra were fitted to model peaks taking into account intrinsic excitations. The measured transition energy of the most intense $\mathrm{KL}_{2} \mathrm{M}_{3}\left({ }^{1} \mathrm{D}_{2}\right)$ Auger line is $7388.1(+/-0.4) \mathrm{eV}$. The obtained relative intensities and energies of the Auger diagram lines are compared to published calculations as well as to experimental data for other $3 d$ transition metals. In addition, the presence of $\mathrm{Ni} 3 \mathrm{~s}, 3 \mathrm{p}$ photoelectron peaks in the spectra excited internally by $\mathrm{Ni} \mathrm{Ka} \mathrm{X-rays} \mathrm{is} \mathrm{shown.}$
\end{abstract}

\section{Introduction}

Owing to the difficulties concerning the high energy resolution measurement and the precise analysis of high-energy Auger electrons spectra there are only a few experimental data on the KLM Auger spectra of $3 \mathrm{~d}$ transition metals ${ }^{1-5}$.

The evaluation of such spectra provides a good opportunity to enhance our knowledge and understanding regarding the underlying physical processes: correlation between the two holes in the final state of the Auger transition (including the coupling of the angular momenta), screening of the charge of the nucleus by inner-shell electrons, rearrangement of the valance electrons in the metallic state because of the sudden appearance of the two holes. In the case of Auger- and photoelectron spectra of transition metals the diagram lines are accompanied by contribution from inelastic electron energy loss which contains broader and less intense structures compared to the free electron like materials. However, the lack of a pronounced plasmon peak does not diminish the importance of the energy loss processes for the determination of the relative intensities of respective lines during the quantitative analysis of the spectra. The measured energy spectrum may contain also fingerprints of shake-up/off processes. The presence of these processes in photoelectron and Auger electron spectra of metallic Ni was shown earlier ${ }^{6-8}$.

In the pioneering work by Asaad and Burhop ${ }^{9}$ results of non-relativistic calculations based on the intermediate angular momentum coupling model were compared with measured data concerning the relative energies and intensities of KLL and KLM Auger lines of a few elements from the atomic number region $Z=26-83$. However, many features of the KLM spectra were difficult to interpret because the quality of the available experimental data was insufficient.

Systematic studies of the KLM Auger spectra of transition metals emitted from radioactive samples were published by Kovalik et al. ${ }^{1-4}$. Némethy et al. ${ }^{5}$ used high energy X-ray radiation for excitation of the spectra of the elements mentioned above. 
In this paper, experimental Auger KLM spectra of metallic Ni measured with considerably improved energy resolution and statistics are presented and evaluated. The results derived from the spectra - the KLM Auger transition energies and intensities related to the $\mathrm{KL}_{2} \mathrm{M}_{3}\left({ }^{1} \mathrm{D}_{2}\right)$ peak - are compared to earlier experimental (for other $3 d$ metals) and theoretical data. In addition, the contributions of the bulk, surface and intrinsic plasmon excitations to the spectral line shape are investigated.

\section{Experimental}

The sample used in this study was a high-purity polycrystalline Ni metal foil of $0.25 \mathrm{~mm}$ thickness, cleaned by Ar ion sputtering. The surface cleanliness was checked by photoemission and only a trace amount of oxygen was found. The measurements were performed at DORIS III (HASYLAB, Hamburg, Germany) using monochromated synchrotron radiation and the Tunable High-Energy XPS instrument at the BW2 beamline ${ }^{10}$. The electron energy analyzer (Scienta SES-200) was set to $150 \mathrm{eV}$ pass energy giving $0.3 \mathrm{eV}$ energy resolution.

Because the kinetic energy of the Ni KLM electrons exceeds the working range of the electron spectrometer, an additional positive bias voltage of $2.5 \mathrm{kV}$ was applied to the sample. By comparing $\mathrm{Au} 4 f$ spectra with and without bias voltage it was verified that the biasing had no effect on the line shape. The kinetic energy scale was calibrated by measuring the energy position of the $A u M_{5} N_{6,7} N_{6,7}$ line obtained from a separate $\mathrm{Au}$ foil on the same sample holder. The kinetic energy of this line was assumed to be $2015.8 \mathrm{eV}^{11}$. The excitation energy for the Ni KLM spectra was set to $9200 \mathrm{eV}$ in order to avoid overlap between the KLM Auger manifold and the Ni L-shell photoelectrons. The X-ray incidence angle was $45^{\circ}$ and the Auger electrons were detected in the direction of the surface normal.

\section{Data evaluation}

In order to accurately evaluate the intrinsic energy distribution and line shape of the KLM manifold, all contributions from other processes to the spectra must be taken into account. After subtracting a linear background which mainly originated from the $\mathrm{Ni} 2 s$ photoelectrons scattered inelastically within the sample, the contribution from multiple inelastic loss processes was removed using the formalism of the Partial Intensity Analysis ${ }^{12}$. According to this method, the inelastic background of the experimental spectrum is assumed to be a sum of contributions from electron energy loss processes attributed to different type of excitations. Collective excitation of free or weakly bound electrons in the bulk or near the surface causes energy losses, described by the probability distributions of electron energy losses for bulk and surface loss processes. Assuming the independence of the bulk and surface loss processes, their effects are removed from the spectrum step by step with the iterative formulae described in detail in Ref. 12. For doing this, one needs to know the probability distribution of the energy lost in a single bulk or surface loss process and in addition the number of electrons taking part $n$ times in the given type (bulk or surface) excitation, i.e. the $n$th order partial intensities.

The partial intensities for the bulk energy loss process were determined by Monte Carlo (MC) simulation of electron trajectories, taking into account both the elastic and inelastic scattering processes. The origins of the trajectories were generated with a uniform probability within a depth of $200 \mathrm{~nm}$ beneath the surface. For the Auger electrons having an energy of $7400 \mathrm{eV}$ an Inelastic Mean Free Path (IMFP) value of $8.2 \mathrm{~nm}^{13}$ and Elastic Mean Free Path (EMFP) of $4.2 \mathrm{~nm}^{14}$ were used. The differential cross section data for elastic electron scattering were obtained from the NIST Standard Reference Data Database $64^{14}$. The dependence of the IMFP on the actual position of the electron (moving within the sample) related to the sample surface (due to the change of the partial contributions of the very different bulk and surface energy loss processes in the proximity of the surface) as well as on the energy losses of the electrons during the transport were neglected. The probability distribution of the energy lost in a single bulk energy loss process was calculated from the 
well-known formula of Lindhard ${ }^{12}$ using the optical data of Palik ${ }^{16}$. According to Penn ${ }^{13}$, the momentum transfer was taken into account using the quadratic dispersion relation ${ }^{15}$.

The Surface Excitation Parameter (SEP) - the average number of surface plasmons excited by a signal electron during a single surface crossing ${ }^{17}$, - was derived using the modified formulae of Oswald ${ }^{17}$ and the material parameter given in Ref. 17. Because surface related processes have no major influence on the spectrum, the SEP turned out to be small and a simple model was used to account for surface effects. The partial intensities for surface loss processes were calculated assuming a Poissonian probability distribution with a parameter equal to the $\mathrm{SEP}^{17}$. The probability of an energy loss during a single excitation process near the surface was approximated by the surface energy loss function $\operatorname{Im}\left(\frac{-1}{\varepsilon+1}\right)$, where the complex dielectric function $\varepsilon$ was calculated from the respective optical data ${ }^{16}$.

After removal of the inelastic background contribution, the peaks in the spectrum show a strong asymmetry. In the next step, this spectrum was fitted with a model spectrum consisting of compound model peaks. A compound model peak consists of an asymmetric Lorentzian component to approximate the effect of the intrinsic electron-hole pair creation as well as the natural and instrumental broadening of the Auger diagram lines, and another asymmetric Lorentzian component to account for the intrinsic collective excitations which occur as a consequence of the sudden appearance of the core hole(s) during the Auger process and other electron energy loss processes like shake up/off excitations.

The shape and the intensity of the intrinsic spectrum obtained after background correction may be interpreted as a result of two major processes. The first is the collective intrinsic excitation of free or loosely bound electrons ("intrinsic plasmon excitation"). According to the previous study ${ }^{23}$ the intensity of this process related to the intensity of the main Auger peak in the KLL Auger spectrum of Ge is about $40 \%$. The second is the $3 d-4 d$ initial state shake excitation upon the ionization of the Kshell. The relative intensity of this satellite to the main peak was found to be approx. $40 \%$ in the KLL Auger spectrum of $\mathrm{Ni}^{8}$.

The results of the spectrum evaluation are summarized in Tables 1-4 and in Figs. 1-3. The indicated uncertainties are the estimates of the standard deviations. In the case of the relative Auger line intensities the error is mainly systematic and depends on the model used for the correction for inelastic background and for describing the effects of intrinsic excitations. According to previous work this systematic error is about $15 \%{ }^{18}$. In the case of the experimental Auger transition energies the main sources of error are the finite instrumental resolution and the uncertainty of the fitting procedure, which is larger for broad and less intense peaks. For peaks lying closely together, the uncertainty of their energy separation and widths can cause a larger uncertainty of the measured relative Auger line intensities.

\section{Results and discussion}

Figure 1 shows the measured and the background corrected spectrum while Fig. 2 shows the result of the evaluation of the background corrected spectrum.

\section{Assignment of the KLM Auger diagram lines}

In previous works, Kovalík et al. ${ }^{1-4}$ and Némethy et al. ${ }^{5}$ used nine peaks to fit the KLM Auger spectra of the $3 \mathrm{~d}$ transition metals. These peaks represent many unresolved components due to the 36 peaks predicted to appear in the KLM spectra according to the intermediate angular momentum coupling model $^{4}$. In the present data the much higher energy resolution and improved statistics revealed 13 
separate structures which were individually fitted. 11 of them belong to the KLM Auger spectrum and 2 additional lines are connected with the spectrum excitation. For the assignment of components of KLM Auger spectrum we used published theoretical calculations of the energy positions and the relative intensities of the Ni KLM diagram lines. Asaad and Burhop performed non-relativistic calculations of the KLM Auger transition energies and intensities for many elements, assuming intermediate coupling ${ }^{9}$. Larkins presented relativistic semi-empirical calculations of KLM Auger transition energies using the intermediate coupling scheme ${ }^{19}$. Non-relativistic calculations for intensities in intermediate coupling scheme were published also by Babenkov et $\mathrm{al}^{20}$. Relativistic calculations were made by Chen at al. but in the jj-coupling scheme ${ }^{21}$.

Due to the good energy resolution the $\mathrm{L}_{3} \mathrm{M}_{45}$ group is clearly seen to be composed of two structures, the separation of which is in a good agreement with theory (Table $1 \mathrm{a}, \mathrm{L}_{3} \mathrm{M}_{45}$ ). The low intensity peak appearing in the $\mathrm{KL}_{2} \mathrm{M}_{23}$ group can be identified as $\mathrm{KL}_{2} \mathrm{M}_{2}\left({ }^{1} \mathrm{~S}_{0}\right)$ peak which is predicted by the intermediate coupling model. Its energy position and measured relative intensity of 9.2 to the ${ }^{1} \mathrm{D}_{2}$ peak are in a reasonable agreement with the theory (Table 1a).

The origin of the small peaks appearing near the $\mathrm{KL}_{3} \mathrm{M}_{1}$ group (denoted by $\mathrm{R} 1$ in Fig. 2), and in the high energy tail of the $\mathrm{KL}_{3} \mathrm{M}_{23}$ group (denoted by R2 in Fig. 2) is not obvious. Their energy positions are not compatible with any calculated multiplet components. i.e. they can hardly be identified as constituents of the $\mathrm{L}_{3} \mathrm{M}_{1}$ and $\mathrm{L}_{3} \mathrm{M}_{23}$ groups predicted by the intermediate coupling model. However, on the basis of their absolute energy position and relative intensity, these peaks can be attributed to the $3 \mathrm{~s}$ and $3 p$ photoelectrons excited internally within the bulk Ni sample by characteristic $\mathrm{Ni} \mathrm{K} \alpha_{1}$ radiation which originates - as a competitive process to the Auger transition - from the radiative relaxation of the vacancy photoexcited in the K-shell (see Table 1b).

\section{Relative Ni KLM Auger transition energies}

Regarding the most intense Ni KLM Auger lines $\left(\mathrm{KL}_{1} \mathrm{M}_{1}, \mathrm{KL}_{1} \mathrm{M}_{23}, \mathrm{KL}_{2} \mathrm{M}_{1}, \mathrm{KL}_{3} \mathrm{M}_{1}, \mathrm{KL}_{3} \mathrm{M}_{2}\left({ }^{3} \mathrm{P}_{2}\right)\right)$ the agreement between the measured energies (relative to the most intense $\mathrm{KL}_{2} \mathrm{M}_{3}{ }^{1} \mathrm{D}_{2}$ line) of the KLM Auger diagram lines and the calculations of Larkins ${ }^{19}$ is good while in the case of the less intense line $\left(\mathrm{KL}_{2} \mathrm{M}_{2}{ }^{1} \mathrm{~S}_{0}\right.$, ) the agreement is reasonable. For those transitions which involve electrons of the $\mathrm{M}_{45}$ subshells the measured relative transition energies are lower- by about $7 \mathrm{eV}$ - than the theoretical values (Table 1a). The measured Ni KLM transition energies follow the trend of the series of experimental data obtained for other transition metals by Némethy ${ }^{5}$ et al. and Kovalík et al ${ }^{1-4}$ (Fig 3a, 3b). From the present data the absolute transition energy of the $\mathrm{KL}_{2} \mathrm{M}_{3}\left({ }^{1} \mathrm{D}_{2}\right)$ line is $7388.1 \mathrm{eV}$, which is $10 \mathrm{eV}$ higher than the value calculated by Larkins ${ }^{19}$. Similar discrepancies were observed for some other elements ${ }^{5}$. They are likely connected with a solid state correction applied in the calculations ${ }^{4}$.

\section{Relative intensities of the Ni KLM Auger lines}

Regarding the relative intensities of the Ni KLM Auger lines there are large differences between the respective values predicted by the different theoretical models (Table 3 ). For example, for the intensity of the $\mathrm{KL}_{3} \mathrm{M}_{23}$ transition the relativistic jj coupling model estimates a factor of 2 higher intensity than the intermediate coupling model presented in Ref. 20. In addition, the former model predicts that this transition is two times more intense than the reference $\mathrm{KL}_{2} \mathrm{M}_{23}$ transition (Table 3). The present experimental data (Table 3 ) are in a good agreement with the intermediate coupling model calculations of Babenkov et al. ${ }^{20}$, confirming its validity for the Ni KLM Auger transitions.

A comparison of the relative intensities of the KLM Auger transitions in $3 d$ transition metals obtained previously by Kovalík et al. ${ }^{1-4}$ and by Némethy et al. ${ }^{5}$, reveals remarkable discrepancies. Also there are marked deviations between the measured and the calculated data as well (Table 4). A possible origin 
of the discrepancies between the measured data may be related to the inherent sensitivity of the derived relative Auger intensities to the background correction procedure and to the intrinsic line shape used in the evaluation ${ }^{18}$. In the case of transition metals, in addition to the 'elastic' photoelectron or Auger peak, inelastic bulk, surface and intrinsic excitations are producing a broad and overlapping energy loss structure which generally is spread over a much wider energy range than in the case of free-electron metals. To consider these features accurately in the numerical modeling is a challenging task. In the present work, the background correction was done using the Partial Intensity Analysis method $^{12}$ in combination with the Monte Carlo simulation of electron trajectories. This is a sophisticated and much more appropriate approach to take into account the effects of multiple elastic and inelastic electron scattering processes along the path of the signal electron within the solid sample $^{22}$.

Despite of the difficulties mentioned above, the measured relative intensities of the KLM Auger diagram lines of metallic $\mathrm{Ni}$ are in a good agreement with the results of the calculations obtained for free atoms in the intermediate coupling scheme (see Table 3, Ref. 20) for closed shells, while in the case of open $\mathrm{M}_{45}$ shells the agreement with relativistic calculations using the $\mathrm{jj}$ coupling scheme (Table 3.) is reasonable. The intensity of the $\mathrm{KL}_{3} \mathrm{M}_{23}$ Auger transition line relative to the intensity of the $\mathrm{KL}_{2} \mathrm{M}_{23}$ transition line is sensitive to the angular momentum coupling model. In the case of the $\mathrm{jj}$ coupling scheme this intensity ratio is 1.95 while in the case of intermediate coupling (Ref. 20) it is 0.97 , in a good agreement with the experimental value of 0.83 obtained in this work.

\section{Ni KLM Auger line widths}

The natural energy widths for the Ni KLM Auger lines were obtained from the spectrum as twice the half width at half maximum measured at the high kinetic energy side of the high energy component of the model peak used for fitting the background corrected spectra. In this way, the strong broadening of the peak at the low kinetic energy side due to the energy losses with intrinsic origin is not taken into account, while the effects of the finite energy resolution of the electron energy analyzer, possible unresolved components are included. The measured quantities are compared to a simple estimate of the Auger line widths (Table 5) obtained from the sum of the recommended values of the energy widths of the atomic levels ${ }^{24}$ involved in the Auger transition. The agreement between the results of the measurement and the theory is good. In the case of $\mathrm{L}_{2} \mathrm{M}_{45}$ and $\mathrm{L}_{3} \mathrm{M}_{45}$ the difference between experiment and theory is larger (Table 5) because of many unresolved components in the data which are predicted by the intermediate coupling model.

\section{Summary}

High-resolution KLM Auger spectra of polycrystalline Ni metal, excited using monochromated synchrotron radiation were measured with high statistical accuracy. The raw experimental data were corrected for the contributions of multiple elastic and inelastic scattering of the electrons within the solid sample by using the Partial Intensity Analysis method ${ }^{12}$. Monte Carlo simulation was applied for the electron trajectories and the influence of the energy loss processes of intrinsic origin was taken into account by the use of compound model peaks in the fitting procedure. The measured kinetic energy of the most intense $\mathrm{KL}_{2} \mathrm{M}_{23}\left({ }^{1} \mathrm{D}_{2}\right)$ peak, the relative transition energies and relative intensities of the KLM Auger peaks were compared with theoretical and experimental data obtained earlier for other $3 d$ transition metals. Due to considerably better energy resolution and higher statistical accuracy, the present work provides experimental data of much higher quality than previous studies ${ }^{1-5}$, clearly identifying additional structure in the fitted spectra which were corrected for the background from multiply scattered electrons. The appearance of the additional peaks can be explained as a consequence of the applicability of the intermediate coupling model for describing the KLM Auger spectra of $\mathrm{Ni}$ as well as a consequence of the excitation of $\mathrm{Ni} 3 s$ and $3 p$ photoelectrons by the characteristic $\mathrm{Ni} \mathrm{K} \alpha_{1}$ radiation originating from the radiative relaxation of the initial vacancy in the Kshell. The relative Ni KLM Auger transition energies determined from the spectra are in a good overall agreement with the relativistic calculations of Larkins ${ }^{19}$ and follow the trends of previous 
measurements of the transition metals $Z=23-26$. Regarding the relative intensities of the Ni KLM Auger lines the present results show a good agreement with the calculations using the intermediate angular momentum coupling scheme $\mathrm{e}^{20}$. The natural energy widths of the Ni Auger KLM lines obtained from the measurement are in a good agreement with the simple theoretical estimates.

\section{Acknowledgements}

The authors are indebted to Prof. W. S. M. Werner for his invaluable support in applying the Partial Intensity Analysis method for inelastic background correction. This work was supported by DESY and the European Community under the contract RII3-CT2004-506008 (IA-SFS).

\section{References:}

[1] A. Kovalik, V. Brabec, J. Novak, O. Dragoun, V. M. Gorozhankin, A. F. Novgorodov, T. Vylov, J. Electron Spectrosc. Relat. Phenom. 50 (1990) 89

[2] A. Kovalik, M. Rysavy, V. Brabec, O. Dragoun, A. Inoyatov, A. F. Novgorodov, T. Vylov, A. Prostakov, Phys. Scr. 37 (1988) 871

[3] A. Kovalik, A. Inoyatov, A. F. Novgorodov, V. Brabec, M. Rysavy, T. Vylov, O. Dragoun, A. Minkova, J. Phys. B 20 (1987) 3997

[4] A. Kovalik, V. M. Gorozhankin, T. Vylov, P. V. Filosofov, N. Coursol, E. A. Zakushev, C. Briancon, A. Minkova, M. Rysavy, O. Dragoun, J. Electron Spectrosc. Relat. Phenom. 95 (1998) 1

[5] A. Némethy, L. Kövér, I. Cserny, D. Varga, P. B. Barna, J. Electron Spectrosc. Relat. Phenom. 82 (1996) 31

[6] D. Briggs and M.P. Seah, Practical Surface Analysis, John Wiley and Sons, (1983), page 131.

[7] S. Hüfner, Photoelectron Spectroscopy ( $3^{\text {rd }}$ Edition), Springer-Verlag, Berlin, 2003

[8] L. Kövér, Z. Berényi, I. Cserny, L. Lugosi, W. Drube, T. Mukoyama, V. R. R. Medicherla, Phys. Rev. B 73 (2006) 195101

[9] W. N. Asaad and E.H.S. Burhop, Proc. Phys. Soc. 71 (1958) 369

[10] W. Drube, H. Schulte-Schrepping, H.-G. Schmidt, R. Treusch, G. Materlik, Rev. Sci. Instrum. 66 (1995) 1668

[11] M.P. Seah, J. Electron Spectrosc. Relat. Phenom. 97 (1998) 235

[12] W. S. M. Werner, Surf. Interface Anal. 31 (2001) 141

[13] D.R. Penn, Phys. Rev. B 35 (1985) 482

[14] Elastic Electron Cross Section Database, Version 2.0 Standard Reference Data Program Database 64, April 2000, NIST, Gaithersburg, MD, USA

[15] R. Shimizu, Z. Ding, Rep. Prog. Phys. 55 (1992) 487

[16] E. D. Palik (Ed.), Handbook of Optical Constants of Solids II. Academic Press New York,1991

[17] W. S. M. Werner, L. Kövér, S. Egri, J. Tóth, D. Varga, Surf. Sci. 585 (2005) 85-94

[18] L. Kövér, S. Egri, Z. Berényi, J. Tóth, I. Cserny, D. Varga, J. Electron Spectrosc. Relat. Phenom. 2007 in press

[19] F. P. Larkins At. Data Nucl Data Tables 20 (1977) 311

[20] M. I. Babenkov, V. C. Zhdanov, Abstracts of 36th. Conf. on Nucl. Spectrosc. Nucl. Structure, Leningrad, Nauka, 1986, p. 268 (in Russian) 
[21] M.H. Chen, B. Crasemann, H. Mark At. Data Nucl. Data Tables 24 (1979) 13

[22] W. S. M. Werner, Surf. Interface Anal. 37 (2005) 846

[23] Z. Berényi, L. Kövér, S. Tougaard, F. Yubero, J. Tóth, I. Cserny, D. Varga, J. Electron Spectrosc. Relat. Phenom. 135 (2004) 177

[24] J. L. Campbell, T. Papp, At. Data Nucl. Data Tabl. 77 (2001)

[25] I. A. Bearden, Rev. Mod. Phys. 39 (1967) 78

[26] J. C. Fuggle and N. Mårtensson J. Electron Spectrosc. Relat. Phenom. 21 (1980) 275

[27] J. H. Scofield, UCRL-51326 Lawrence Livermore Laboratory, University of California, Livermore, California (1973) 


\section{Figure captions}

Fig.1. The measured (upper) and background corrected (lower) KLM Auger spectra photoexcited from polycrystalline Ni metal.

Fig. 2. The KLM Auger spectrum photoexcited from polycrystalline Ni metal, (inelastic background removed) with the component peaks and the result of the fit (solid lines).

Fig. 3a. The measured KLM Auger energies of $3 d$ transition metals for transitions $\mathrm{KL}_{1} \mathrm{M}_{1}$, $\mathrm{KL}_{1} \mathrm{M}_{23}, \mathrm{KL}_{1} \mathrm{M}_{45}, \mathrm{KL}_{2} \mathrm{M}_{1}, \mathrm{KL}_{3} \mathrm{M}_{1}$ (denoted by different markers) relative to the energy of the prominent $\mathrm{KL}_{2} \mathrm{M}_{23}\left({ }^{1} \mathrm{D}_{2}\right)$ line (hollow triangles at the top).

Fig. 3b. Same as Fig. $3 \mathrm{a}$ for transitions $\mathrm{KL}_{2} \mathrm{M}_{23}, \mathrm{KL}_{2} \mathrm{M}_{45}, \mathrm{KL}_{3} \mathrm{M}_{45}$ (denoted by different markers). 
Table 1a

Ni KLM Auger transition energies relative to the most intense $K_{2} M_{3}\left({ }^{1} D_{2}\right)$ line

\begin{tabular}{|c|c|c|c|}
\hline \multicolumn{2}{|c|}{ Transition } & \multirow{2}{*}{$\begin{array}{c}\text { Theory [19] } \\
-182.80\end{array}$} & \multirow{3}{*}{$\begin{array}{c}\text { Present } \\
\text { Measurement } \\
-184.0(1.0)^{c}\end{array}$} \\
\hline \multirow{2}{*}{$\mathrm{KL}_{1} \mathrm{M}_{1}$} & ${ }^{1} S_{0}(18.4)$ & & \\
\hline & ${ }^{3} S_{1}(0.1)$ & -177.10 & \\
\hline \multirow{2}{*}{$\mathrm{KL}_{1} \mathrm{M}_{3}$} & ${ }^{3} P_{2}(2.9)$ & -134.20 & \multirow{4}{*}{$-138.0(1.0)$} \\
\hline & ${ }^{3} \mathrm{P}_{1}(4.9)$ & -134.20 & \\
\hline \multirow{2}{*}{$\mathrm{KL}_{1} \mathrm{M}_{2}$} & ${ }^{3} P_{0}(0.6)$ & -134.20 & \\
\hline & ${ }^{1} P_{1}(18.6)$ & -133.80 & \\
\hline \multirow{2}{*}{$\mathrm{KL}_{1} \mathrm{M}_{4}$} & ${ }^{1} D_{2}(0.0)$ & -62.80 & \multirow{4}{*}{$-6.8(2.0)$} \\
\hline & ${ }^{3} D_{1}(0.06)$ & -61.30 & \\
\hline \multirow{2}{*}{$\mathrm{KL}_{1} \mathrm{M}_{5}$} & ${ }^{3} D_{3}(0.12)$ & -61.30 & \\
\hline & ${ }^{3} D_{2}(0.06)$ & -61.30 & \\
\hline \multirow{2}{*}{$\mathrm{KL}_{2} \mathrm{M}_{1}$} & ${ }^{1} \mathrm{P}_{1}(14.2)$ & -44.10 & \multirow{2}{*}{$-42.4(1.5)$} \\
\hline & ${ }^{3} \mathrm{P}_{0}(0.5)$ & -43.20 & \\
\hline \multirow{2}{*}{$\mathrm{KL}_{3} \mathrm{M}_{1}$} & ${ }^{3} \mathrm{P}_{1}(11.0)$ & -27.50 & \multirow{2}{*}{$-26.6(0.5)$} \\
\hline & ${ }^{3} \mathrm{P}_{2}(2.4)$ & -26.00 & \\
\hline $\mathrm{KL}_{2} \mathrm{M}_{2}$ & ${ }^{1} S_{0}(7.9)$ & -3.50 & $-5.8(2.0)$ \\
\hline $\mathrm{KL}_{2} \mathrm{M}_{3}$ & ${ }^{1} D_{2}(100)$ & 0.00 & \multirow{3}{*}{0} \\
\hline $\mathrm{KL}_{2} \mathrm{M}_{2}$ & ${ }^{3} P_{1}(0.00)$ & 0.90 & \\
\hline $\mathrm{KL}_{2} \mathrm{M}_{3}$ & ${ }^{3} D_{1}(0.1)$ & 3.60 & \\
\hline $\mathrm{KL}_{3} \mathrm{M}_{3}$ & ${ }^{3} P_{0}(2.5)$ & 12.70 & \multirow{6}{*}{$15.0(0.4)$} \\
\hline \multirow{2}{*}{$\mathrm{KL}_{3} \mathrm{M}_{2}$} & ${ }^{3} P_{2}(24.1)$ & 15.60 & \\
\hline & ${ }^{3} S_{1}(0.1)$ & 18.10 & \\
\hline \multirow{3}{*}{$\mathrm{KL}_{3} \mathrm{M}_{3}$} & ${ }^{3} D_{2}(1.6)$ & 18.40 & \\
\hline & ${ }^{3} D_{3}(0.1)$ & 20.60 & \\
\hline & ${ }^{1} \mathrm{P}_{1}(0.0)$ & 21,00 & \\
\hline $\mathrm{KL}_{2} \mathrm{M}_{4}$ & ${ }^{3} D_{1}$ & 73.50 & \multirow{4}{*}{$68.8(1.0)$} \\
\hline $\mathrm{KL}_{2} \mathrm{M}_{5}$ & ${ }^{3} \mathrm{~F}_{3}$ & 74.00 & \\
\hline $\mathrm{KL}_{2} \mathrm{M}_{4}$ & ${ }^{3} \mathrm{P}_{2}$ & 74.30 & \\
\hline $\mathrm{KL}_{2} \mathrm{M}_{5}$ & ${ }^{3} \mathrm{~F}_{2}$ & 75.40 & \\
\hline \multirow{3}{*}{$\mathrm{KL}_{3} \mathrm{M}_{4}$} & ${ }^{1} \mathrm{P}_{1}$ & 90.00 & \multirow{5}{*}{$83.9(1.0)$} \\
\hline & ${ }^{1} \mathrm{~F}_{3}$ & 90.40 & \\
\hline & ${ }^{3} P_{0}$ & 90.90 & \\
\hline $\mathrm{KL}_{3} \mathrm{M}_{5}$ & ${ }^{3} \mathrm{P}_{1}$ & 91.20 & \\
\hline $\mathrm{KL}_{3} \mathrm{M}_{4}$ & ${ }^{3} D_{2}$ & 91.50 & \\
\hline \multirow{3}{*}{$\mathrm{KL}_{3} \mathrm{M}_{5}$} & ${ }^{3} D_{3}$ & 92.10 & \multirow{3}{*}{$86.5(1.0)$} \\
\hline & ${ }^{3} \mathrm{~F}_{4}$ & 92.60 & \\
\hline & ${ }^{1} \mathrm{D}_{2}$ & 92.70 & \\
\hline $\mathrm{KL}_{2} \mathrm{M}_{23}$ & ${ }^{17} D_{2}$ & 7378.4 & $7388.1(0.4)$ \\
\hline
\end{tabular}

${ }^{\mathrm{c}}-184.4(1.0)$ is a short notation for $-184.4 \pm 1.0$. The number in brackets following the symbol of the transition gives the relative intensity of the corresponding peak in the Auger spectrum according to the nonrelativistic calculations of Asaad et al.. 


\section{Table 1b}

The absolute energy and intensity ratio of the $R_{1}, R_{2}$ lines (see Fig. 2). The theoretical values were calculated using refs. $[25,26,27]$

\begin{tabular}{|c|c|c|c|}
\hline peaks & Measurement $/ \mathrm{eV}$ & \multicolumn{2}{|c|}{ Theory } \\
\hline $\mathrm{R}_{1}$ & $7366.8(0.5)$ & \multicolumn{2}{|c|}{$7367.35(\mathrm{Ni} 3 s / \mathrm{Ni} \mathrm{K \alpha} 1)$} \\
\hline $\mathrm{R}_{2}$ & $7411.5(0.5)$ & $\begin{array}{c}7411.95 \\
\left(\mathrm{Ni} 3 p_{1 / 2} / \mathrm{Ni} \mathrm{K \alpha} 1\right)\end{array}$ & $\begin{array}{c}7410.15 \\
\left(\mathrm{Ni} 3 p_{3 / 2} / \mathrm{Ni} \mathrm{K \alpha} 1\right)\end{array}$ \\
\hline Int. $\mathrm{R}_{1} / \mathrm{R}_{2}$ & $2.6(1.0)$ & \multicolumn{2}{|c|}{1.4} \\
\hline
\end{tabular}




\section{Table 2}

KLM Auger transition energies (in $\mathrm{eV}$ ) of $3 d$ transition metals relative to the most intense $\mathrm{KL}_{2} \mathrm{M}_{3}\left({ }^{1} \mathrm{D}_{2}\right)$ transition. Data for $\mathrm{V}, \mathrm{Cr}, \mathrm{Mn}, \mathrm{Fe}$ were taken from Ref.[5], while in the cases denoted by (k) from Ref. [4]

\begin{tabular}{|c|c|c|c|c|c|}
\hline Transition & V & $\mathrm{Cr}$ & $\mathrm{Mn}$ & $\mathrm{Fe}$ & $\mathrm{Ni}$ (present work) \\
\hline $\begin{array}{c}\mathrm{KL}_{1} \mathrm{M}_{1} \\
\left({ }^{1} \mathrm{~S}_{0}\right)\end{array}$ & $\begin{array}{c}-135.2 \\
(0.5)\end{array}$ & $\begin{array}{c}-155.3 \\
(2.1)\end{array}$ & $\begin{array}{c}-156.6 \\
(0.8)\end{array}$ & $\begin{array}{c}-165.0(\mathrm{k}) \\
(0.9)\end{array}$ & $-184.4(1.0)$ \\
\hline $\begin{array}{c}\mathrm{KL}_{1} \mathrm{M}_{23} \\
\left({ }^{1} \mathrm{P}_{1}\right)\end{array}$ & $\begin{array}{c}-107.1 \\
(0.5)\end{array}$ & $\begin{array}{c}-116.1 \\
(1.0)\end{array}$ & $\begin{array}{c}-118.3 \\
(1.3)\end{array}$ & $\begin{array}{c}-123.5(\mathrm{k}) \\
(0.8)\end{array}$ & $-137.9(1.0)$ \\
\hline $\begin{array}{c}\mathrm{KL}_{1} \mathrm{M}_{45} \\
\left({ }^{3} \mathrm{D}_{3}\right)\end{array}$ & $\begin{array}{r}-74.7 \\
(0.5)\end{array}$ & $\begin{array}{c}-65.5 \\
(1.6)\end{array}$ & $\begin{array}{r}-67.2 \\
(1.4)\end{array}$ & $\begin{array}{c}-63.5(\mathrm{k}) \\
(1.2)\end{array}$ & $-69.8(2.0)$ \\
\hline $\begin{array}{l}\mathrm{KL}_{2} \mathrm{M}_{1} \\
\left({ }^{1} \mathrm{P}_{1}\right)\end{array}$ & - & $\begin{array}{r}-34.7 \\
(0.7)\end{array}$ & $\begin{array}{l}-33.3 \\
(1.1)\end{array}$ & $\begin{array}{r}-38.0 \\
(0.2)\end{array}$ & $-42.4(1.5)$ \\
\hline $\begin{array}{c}\mathrm{KL}_{3} \mathrm{M}_{1} \\
\left({ }^{3} \mathrm{P}_{1}\right)\end{array}$ & - & $\begin{array}{l}-21.1 \\
(1.3)\end{array}$ & $\begin{array}{l}-21.7 \\
(0.8)\end{array}$ & $\begin{array}{l}-27 \\
(1.0)\end{array}$ & $-26.7(0.5)$ \\
\hline $\begin{array}{c}\mathrm{KL}_{2} \mathrm{M}_{23} \\
\left({ }^{1} \mathrm{D}_{2}\right)\end{array}$ & 0 & 0 & 0 & 0 & 0 \\
\hline $\begin{array}{c}\mathrm{KL}_{3} \mathrm{M}_{23} \\
\left({ }^{3} \mathrm{P}_{2}\right)\end{array}$ & $\begin{array}{c}7.6 \\
(0.2)\end{array}$ & $\begin{array}{c}6.7 \\
(0.6)\end{array}$ & $\begin{array}{c}9.6 \\
(0.7)\end{array}$ & $\begin{array}{l}11.0 \\
(0.7)\end{array}$ & $15.0(0.4)$ \\
\hline $\begin{array}{c}\mathrm{KL}_{2} \mathrm{M}_{45} \\
\left({ }^{3} \mathrm{~F}_{3}\right)\end{array}$ & $\begin{array}{l}40.6 \\
(0.4)\end{array}$ & $\begin{array}{l}26.5 \\
(1.1)\end{array}$ & $\begin{array}{l}48.8 \\
(0.9)\end{array}$ & $\begin{array}{l}54.6 \\
(0.5)\end{array}$ & $68.8(1.0)$ \\
\hline $\begin{array}{c}\mathrm{KL}_{3} \mathrm{M}_{45} \\
\left({ }^{3} \mathrm{P}_{1}\right)\end{array}$ & $\begin{array}{l}46.9 \\
(0.5)\end{array}$ & $\begin{array}{l}50.9 \\
(0.8)\end{array}$ & $\begin{array}{l}60.5 \\
(0.9)\end{array}$ & $\begin{array}{l}66.6 \\
(0.5)\end{array}$ & $86.5(1.0)$ \\
\hline
\end{tabular}


Table 3

Ni KLM Auger line intensities relative to the most intense $K_{2} M_{3}$ $\left({ }^{1} D_{2}\right)$ peak compared to theoretical values

\begin{tabular}{|ccccc|}
\hline Transition & $\begin{array}{c}\text { Present } \\
\text { work (exp.) }\end{array}$ & $\begin{array}{c}\text { jj coupling } \\
\text { relativistic } \\
{[21]}\end{array}$ & $\begin{array}{c}\text { Int. coupling } \\
{[20]}\end{array}$ & $\begin{array}{c}\text { Int. coupling } \\
{[9]}\end{array}$ \\
\hline $\mathrm{KL}_{1} \mathrm{M}_{1}$ & $26(4.0)$ & 46 & 25 & 17 \\
$\mathrm{KL}_{1} \mathrm{M}_{23}$ & $52(8.0)$ & 70 & 42 & 25 \\
$\mathrm{KL}_{1} \mathrm{M}_{45}$ & $9(1.0)$ & 4 & & 0.2 \\
$\mathrm{KL}_{2} \mathrm{M}_{1}$ & $19(3.0)$ & 22 & 15 & 14 \\
$\mathrm{KL}_{3} \mathrm{M}_{1}$ & $27.5(4.0)$ & 40 & 23 & 12 \\
$\mathrm{KL}_{2} \mathrm{M}_{23}$ & $100(15.0)$ & 100 & 100 & 100 \\
$\mathrm{KL}_{3} \mathrm{M}_{23}$ & $83(12.0)$ & 195 & 97 & 26 \\
$\mathrm{KL}_{2} \mathrm{M}_{45}$ & $9(1.0)$ & 7 & & 0 \\
$\mathrm{KL}_{3} \mathrm{M}_{45}$ & $17(3.0)$ & 14 & & 0 \\
\hline
\end{tabular}




\section{Table 4}

Relative intensites of KLM Auger diagram lines of Ni (present work) and the respective previously measured $\mathrm{V}, \mathrm{Cr}, \mathrm{Mn}, \mathrm{Fe}$ by Némethy et al. [5] and Kovalík et al. [4] (denoted by (k) ). Results of calculations in the intermediate coupling scheme Ref. [20] and jj coupling scheme Ref. [21]. (jj values are quoted where intermediate coupling data are are unavailable).

\begin{tabular}{|c|c|c|c|c|c|c|c|c|}
\hline Transition & $\begin{array}{c}\text { Theory } \\
Z=20\end{array}$ & ${ }^{23} \mathrm{~V}$ & ${ }^{24} \mathrm{Cr}$ & ${ }^{25} \mathrm{Mn}$ & $\begin{array}{c}\text { Theory } \\
Z=25\end{array}$ & ${ }^{26} \mathrm{Fe}$ & ${ }^{28} \mathrm{Ni}$ & $\begin{array}{l}\text { Theory } \\
Z=30\end{array}$ \\
\hline $\mathrm{KL}_{1} \mathrm{M}_{1}$ & 20 & $\begin{array}{l}11.0 \\
(1.4)\end{array}$ & $\begin{array}{l}58.8 \\
(7.5)\end{array}$ & $\begin{array}{l}23.7 \\
(2.0)\end{array}$ & 24 & $\begin{array}{l}27(\mathrm{k}) \\
(1.0)\end{array}$ & $\begin{array}{c}26 \\
(4.0)\end{array}$ & 27 \\
\hline $\mathrm{KL}_{1} \mathrm{M}_{23}$ & 30 & $\begin{array}{l}17.1 \\
(6.5)\end{array}$ & $\begin{array}{l}38.1 \\
(4.2)\end{array}$ & $\begin{array}{l}33.3 \\
(1.5)\end{array}$ & 38 & $\begin{array}{l}42(\mathrm{k}) \\
(2.0)\end{array}$ & $\begin{array}{c}52 \\
(8.0)\end{array}$ & 44 \\
\hline $\mathrm{KL}_{1} \mathrm{M}_{45}$ & $0(\mathbf{j j})$ & $\begin{array}{c}2.7 \\
(0.5)\end{array}$ & $\begin{array}{c}6.7 \\
(1.5)\end{array}$ & $\begin{array}{c}5.3 \\
(1.1)\end{array}$ & $1.4(\mathrm{jj})$ & $\begin{array}{l}4(\mathrm{k}) \\
(1.0)\end{array}$ & $\begin{array}{c}9 \\
(1.0)\end{array}$ & $4(\mathrm{jj})$ \\
\hline $\mathrm{KL}_{2} \mathrm{M}_{1}$ & 14 & $\begin{array}{c}5.2(\mathrm{k}) \\
(0.3)\end{array}$ & $\begin{array}{l}15.0 \\
(1.6)\end{array}$ & $\begin{array}{c}4.7 \\
(1.7)\end{array}$ & 14 & $\begin{array}{c}4.4 \\
(0.4)\end{array}$ & $\begin{array}{c}19 \\
(3.0)\end{array}$ & 15 \\
\hline $\mathrm{KL}_{3} \mathrm{M}_{1}$ & 15 & $\begin{array}{l}36.4 \\
(0.8)\end{array}$ & $\begin{array}{l}47.2 \\
(5.5)\end{array}$ & $\begin{array}{l}25.8 \\
(1.6)\end{array}$ & 21 & $\begin{array}{l}16.4 \\
(0.8)\end{array}$ & $\begin{array}{l}27.5 \\
(4.0)\end{array}$ & 25 \\
\hline $\mathrm{KL}_{2} \mathrm{M}_{23}$ & 100 & 100 & 100 & 100 & 100 & $\begin{array}{c}100 \\
(4.0)\end{array}$ & $\begin{array}{c}100 \\
(15.0)\end{array}$ & 100 \\
\hline $\mathrm{KL}_{3} \mathrm{M}_{23}$ & 38 & $\begin{array}{c}8.9 \\
(1.9)\end{array}$ & $\begin{array}{l}46.1 \\
(6.7)\end{array}$ & $\begin{array}{l}31.3 \\
(2.4)\end{array}$ & 83 & $\begin{array}{l}54.6 \\
(3.6)\end{array}$ & $\begin{array}{c}84 \\
(12.0)\end{array}$ & 108 \\
\hline $\mathrm{KL}_{2} \mathrm{M}_{45}$ & $0(\mathrm{jj})$ & $\begin{array}{c}2.5 \\
(0.2)\end{array}$ & $\begin{array}{c}8.3 \\
(1.5)\end{array}$ & $\begin{array}{c}4.9 \\
(0.9)\end{array}$ & $2.6(\mathrm{jj})$ & $\begin{array}{c}4.2 \\
(0.6)\end{array}$ & $\begin{array}{c}9 \\
(1.0)\end{array}$ & $7(\mathbf{j j})$ \\
\hline $\mathrm{KL}_{3} \mathrm{M}_{45}$ & $0(\mathrm{jj})$ & $\begin{array}{c}3.1 \\
(0.2)\end{array}$ & $\begin{array}{c}9.0 \\
(1.1)\end{array}$ & $\begin{array}{c}5.9 \\
(0.9)\end{array}$ & 8.4(jj) & $\begin{array}{c}5.7 \\
(2.8)\end{array}$ & $\begin{array}{c}17 \\
(3.0)\end{array}$ & $14(\mathrm{jj})$ \\
\hline
\end{tabular}




\section{Table 5}

\section{Energy widths of the KLM Auger lines photoexcited from Ni metal (eV)}

\begin{tabular}{|ccc|}
\hline Transition & $\begin{array}{c}\text { Present work } \\
\text { (exp.) }\end{array}$ & $\begin{array}{c}\text { Theory (sum of natural level widths) } \\
{[24]}\end{array}$ \\
\hline $\mathrm{KL}_{1} \mathrm{M}_{1}\left({ }^{1} \mathrm{~S}_{0}\right)$ & $10.8(0.5)$ & 10.1 \\
$\mathrm{KL}_{1} \mathrm{M}_{23}$ & $9.4(0.5)$ & 9.1 \\
$\mathrm{KL}_{1} \mathrm{M}_{45}$ & $8.4(0.5)$ & 7.8 \\
$\mathrm{KL}_{2} \mathrm{M}_{1}$ & $6.4(0.3)$ & 4.7 \\
$\mathrm{KL}_{3} \mathrm{M}_{1}$ & $5.4(0.3)$ & 4.2 \\
$\mathrm{KL}_{2} \mathrm{M}_{23}\left({ }^{1} \mathrm{D}_{2}\right)$ & $4.4(0.2)$ & 3.7 \\
$\mathrm{KL}_{3} \mathrm{M}_{23}$ & $4.0(0.2)$ & 3.2 \\
$\mathrm{KL}_{2} \mathrm{M}_{45}$ & $4.8(0.2)$ & 2.4 \\
$\mathrm{KL}_{3} \mathrm{M}_{45}(\mathrm{a})^{\mathrm{c}}$ & $1.6(0.2)$ & 1.9 \\
$\mathrm{KL}_{3} \mathrm{M}_{45}(\mathrm{~b})$ & $6.0(0.4)$ & 1.9 \\
\hline
\end{tabular}

${ }^{\mathrm{c}}$ The two components applied to fit the Ni Auger $\mathrm{L}_{3} \mathrm{M}_{45}$ group denoted by $a, b$; see in Fig 2 . 


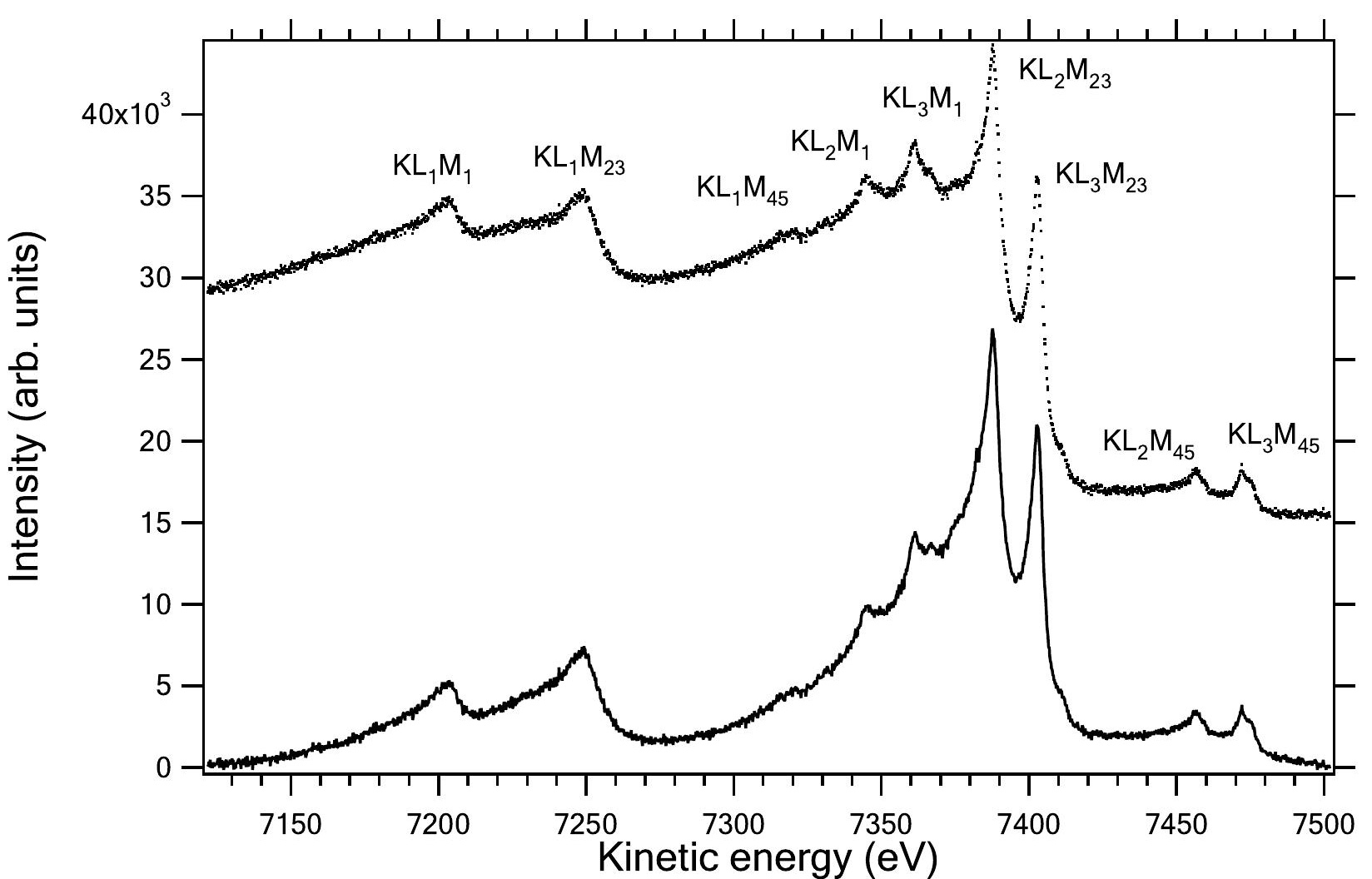

Figure

Figure

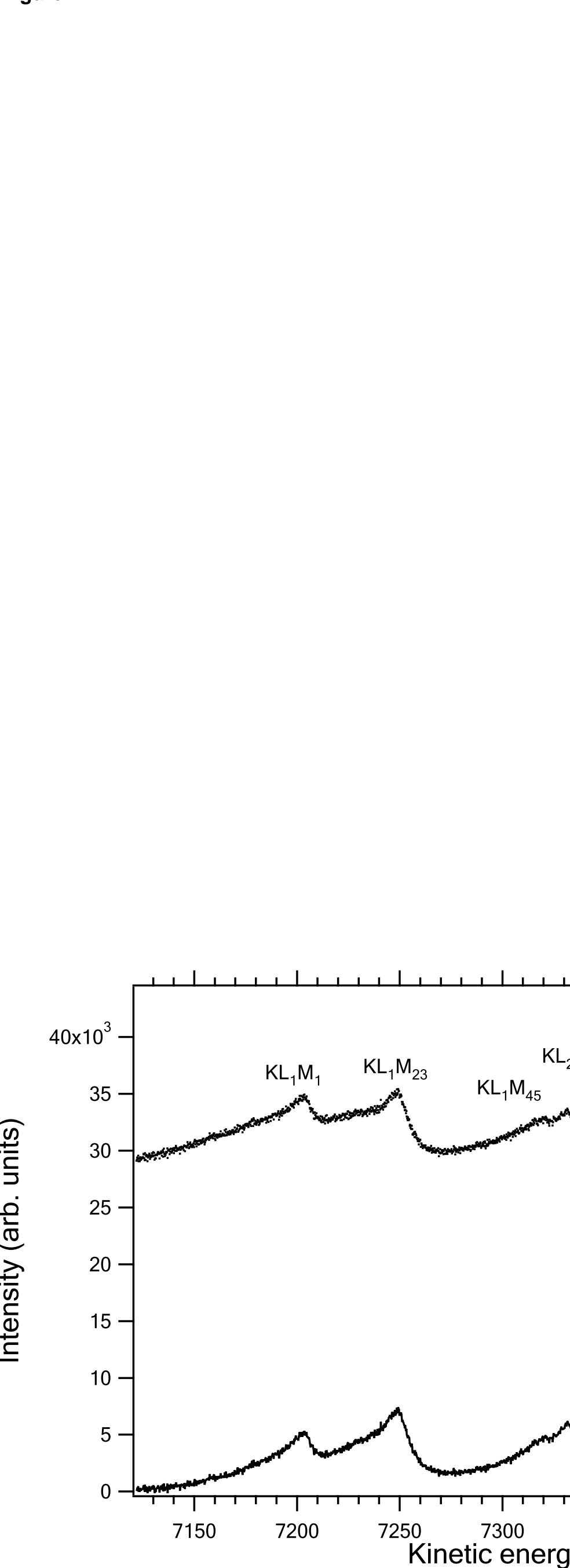

.




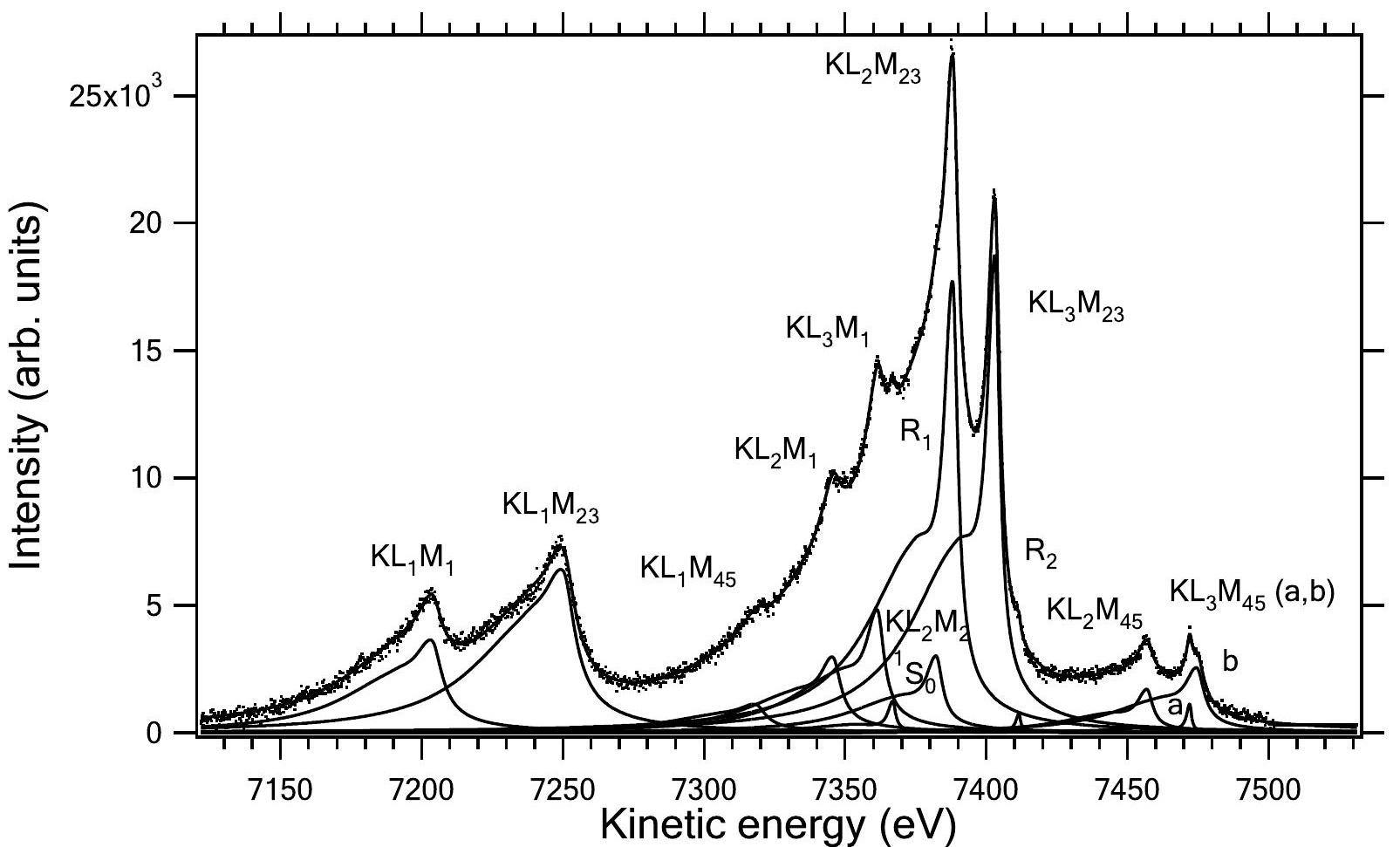


